\title{
SIMILARITY IN SEDIMENT TRANSPORT DUE TO WAVES
}

\author{
S. Yalin, Dozent, Dr.-Ing.,
}

R. C. H. Russell, M.A., A.M.I.C.E., A.M.I.Mech.E. Hydraulics Research Station, Wallingford, England.

\section{ABSTRACT}

The paper concerns the movement by waves of cohesionless sediment lying on a horizontal bed. In particular it concerns the number of dimensionless parameters that are necessary to define the 2-phase motion at the bed; the specification of which would enable perfect similarity to be obtained.

It is shown that in general four dimensionless parameters are necessary; but that when the motion of the water at the bed can be adequately defined by an orbit length ( $a$ ) and a period ( $T$ ), the two-phase motion can be described by the numerical value of three dimensionless parameters. This condition is satisfied when the wave-height is low, because then the orbital motion at the bed is sinusoidal and the drift velocity is negligible.

Model and prototype experiments were conducted in a wave channel, using low waves, in which the scale for depth of water and for wavelengths was $\frac{3}{10}$. The dependent parameters, three of which are sufficient to verify similarity of all aspects of the phenomenon were chosen to be ripple height, ripple length and transport of sediment. The identity of the dimensionless numbers signifying the ripple height, ripple length and transport in model and prototype, shown in Figs. 8,9 and 11 , is proof that similarity had been obtained.

\section{1) DEFINITION OF THE TWO-PHASE PHENOMENON, CHARACTERISTIC PARAMETERS}

Consider two-dimensional progressive wave motion of a real fluid with a finite depth $(i, e, h<I / 2)$ over a horizontal and cohesionless movable bed (Fig. 1). If the motion of the fluid is able to produce the movement of the bed material then both motions depending reciprocally each on the other together constitute an inseparable mechanical whole - a "two-phase phenomenon".

We assume that the following geometrical properties (independent of the absolute size) are specified: 


\section{COASTAL ENGINEERING}

(i) the form of the waves ( $i$, we assume that the function $\mathrm{y} / \mathrm{H}=\varphi(\mathrm{x} / \mathrm{L})$ has a certain definite form; where the $x$-axis travels on the still surface of the fluid with the velocity of wave propagation $C$ ).

(ii) the form of the particle - size distribution curve of the bed material.

(iii) the form of the particles of the bed material.

In this case the two-phase phenomenon in the vicinity of the bed is completely defined by specifying the following 7 independent quantities (characteristic parameters)

$\rho$ density of fluid

$\nu$ kinematic viscosity

$D$ any typical diameter of

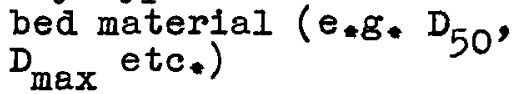

$\gamma_{s}$ specific weight of the bed material in fluid

$a_{b}$ the orbit length at the bed ${ }^{*}$ )

T period

$w_{b}$ the drift velocity at the bed physical properties of fluid

properties of bed material

mechanical properties of the fluid motion at the bed

Hence any mechanical quantity A related to the two-phase pnenomenon in the vicinity of the bed (regardless of whether $A$ is a property of the fluid motion or of the motion of the bed material) must be a certain function of the characteristic parameters ( 1 )

$$
A=f_{A}\left(P, \nu, D, \gamma_{s}, T, a_{b}, w_{b}\right)
$$

The subscript $A$ in $f_{A}$ indicates that the form of the above function varies as $A$, the quantity under investigation, varies.

*) "at the bed" means "at the region just above the boundary layer". Since the distributions of a and with depth both tend to be vertical as they approach the boundary layer more precise definition of location is not necessary. 
SIMILARITY IN SEDIMENT TRANSPORT

DUE TO WAVES

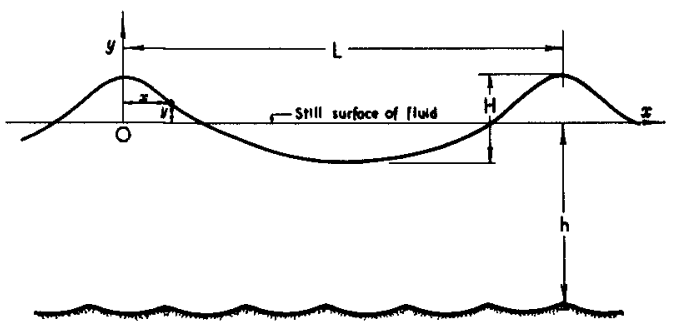

F1g. 1

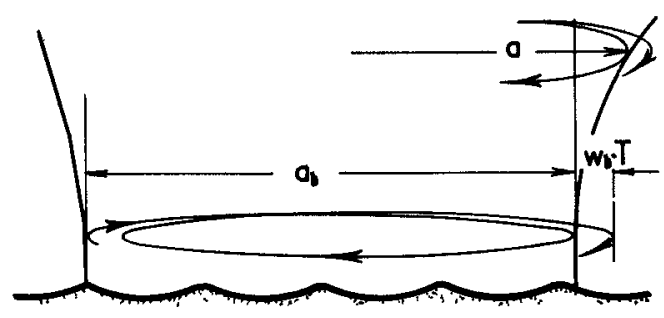

F1g. 2

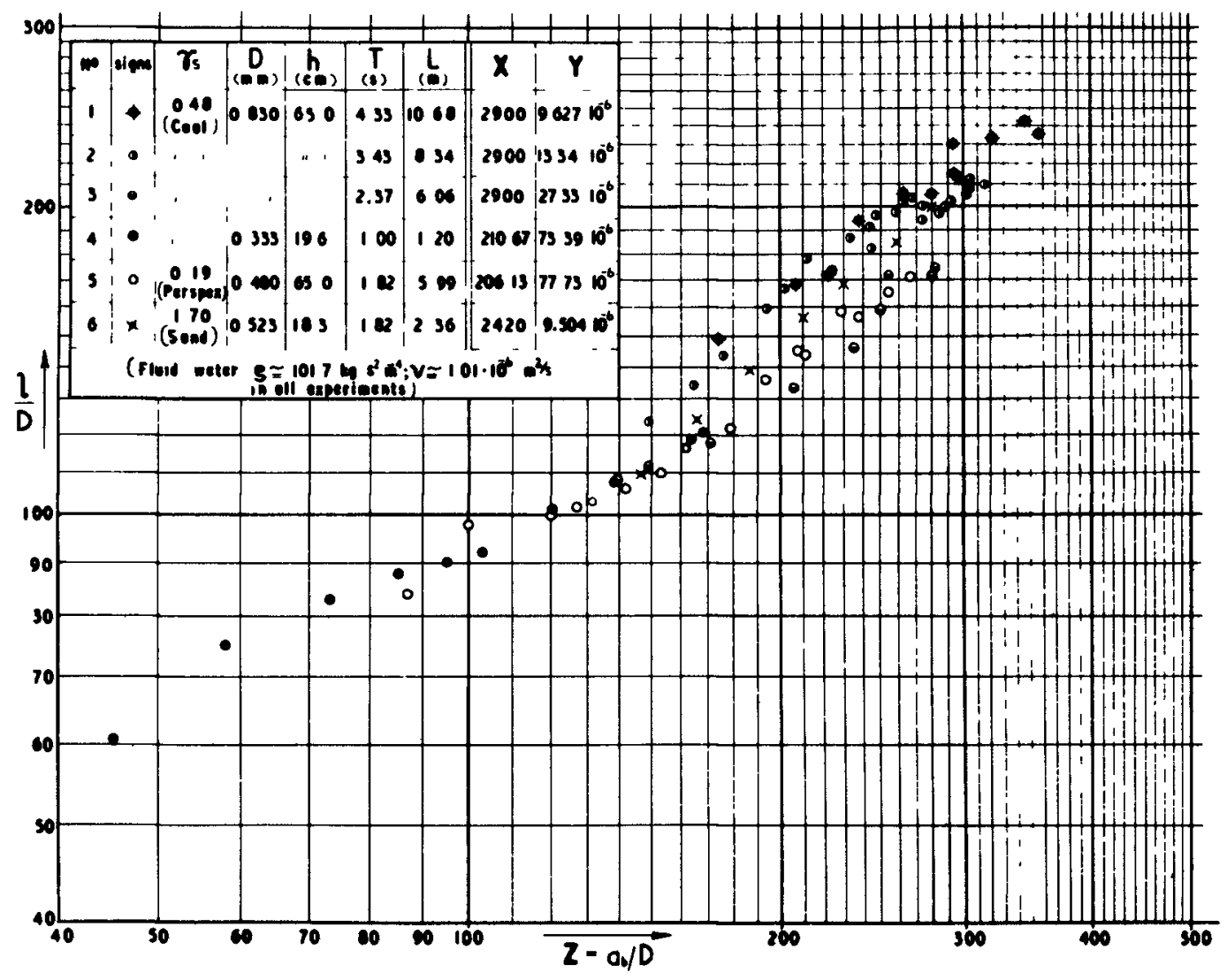

F1g. 3 


\section{COASTAL ENGINEERING}

\section{2) DIMENSIONLESS EXPRESSION OF THE PHENOMENON}

According to the theory of dimensions each dimensional relationship (2) can always be expressed in a dimensionless form as follows

$$
\Pi_{A}=\varphi_{A}(X, Y, Z, W)
$$

where the four variables $X, Y, Z, W$ represent the following dimensionless combinations of 7 dimensional parameters $(1)^{*}$;

$$
X=\frac{\gamma_{s} D^{3}}{P \nu^{2}} ; Y=\frac{\rho \cdot D}{\gamma_{s} T^{2}} ; Z=\frac{a_{b}}{D} ; W=\frac{w_{b} T}{D}
$$

while the function $\Pi_{A}$ is the dimensionless power product

$$
\Pi_{A}=A \cdot D^{x} \gamma_{s}^{y} T^{z}
$$

e.g. If $A=1$ ripple length $\Pi_{1}=1 \cdot D^{-1}=\varphi_{1}(X, Y, Z, W)$

$$
A=\Delta \text { ripple height } \prod_{\Delta}=\Delta D^{-1}=\varphi_{\Delta}(X, Y, Z, W)
$$

*) Selecting $\rho_{N}, T, a_{b}$ as basic quantities we obtain from the

$$
\bar{X}=\frac{2 a_{b} D}{T \cdot \nu} ; \quad \bar{Y}=\frac{\rho \cdot a_{b}}{\gamma_{s} T^{2}} ; \quad \bar{Z}=\frac{a_{b}}{D} ; \quad \bar{W}=\frac{w_{b} T}{a_{b}}
$$

Considering that $2 \cdot a_{b} / T=U$ (mean orbital velocity) and $W_{b} T$ is the distance of transiation of the orbit during the period $T$, it is realized that $\bar{X}$ is a "grain-size Reynolds number", $\bar{Y}$ characterizes the "ratio of the drag force to the weight of the bed material", $\bar{z}$ is "initial relative roughness (or smoothness) of the bed" and $\bar{W}$ is the "relative translation of the orbit due to drift" . However, these distinctly interpretable basic combinations are inappropriate for experimental purposes (e.8* variation in the orbit length $a_{b}$, the easiest case experimentally, lead to variations in each of the dimensionless variables, $\bar{X}, \bar{Y}$ $\bar{Z}, \bar{W}$. Since according to the theory of dimensions any set of dimensionless variables can be replaced by any independent combinations of them, the variables (4) have been chosen as the following independent combinations of $\bar{X}, \bar{Y}$, $\bar{Z}, \bar{W} . \quad X=\bar{X}^{2} \bar{Y}^{-1} \bar{Z}^{-1} \frac{1}{4}, \quad Y=\bar{Y} \cdot \bar{Z}^{-1}, \quad Z=\bar{Z} \quad ; \quad W=\bar{W} \bar{Z}$ (See for "basic quantities", "J=Theorem", "independent com binations" etc. in Ref.[1]) 


\section{SIMILARITY IN SEDIMENT TRANSPORT DUE TO WAVES}

$A=G$ the weight of the transported bed
material (during a certain time $t)$
$\Pi_{G}=G D^{-3} \gamma_{S}^{-1}=\varphi_{G}(X, Y, Z, W)$ etc.

If the wave-height is "small" in comparison to the wave-length ("Waves with small amplitude") then the influence of the variable $W$ is negligible, and thus the general form (3) can be assumed to be a function of 3 variables only:

$$
\Pi_{A}=\varphi_{A}(X, Y, Z)
$$

For example, in this case the expression of ripple length (6) becomes

$$
\Pi_{1}=\frac{1}{\mathrm{D}}=\varphi_{1}(\mathrm{X}, \mathrm{Y}, \mathrm{Z})
$$

which is the "family of the surfaces", or the "family of the family of curves". Putting $X=$ const we obtain from (10) the following function of 2 variables only

$$
\frac{1}{\bar{D}}=\Phi_{1}(\text { const, } Y, Z)=\bar{\Phi}_{1}(Y, Z)
$$

which can be represented by a "family of curves". If in addition, $Y=$ const, then $I / D$ is a function of one variable only; and consequently can be represented by a "single curve":

$$
\left.\frac{1}{D}=\varphi_{1} \text { (const, const, } \mathrm{z}\right)=\overline{\bar{\Phi}_{1}}(\mathrm{z})
$$

(This explains why, in plotting 1 versus $a_{b}$ (which, in dimensionless plotting, implies (12)), the experimental points are so scattered (see for example ref. [2]) ${ }^{*}$. This is not only because the two lengths 1 and $a b$ have not been measured properly, but also because it was attempted to represent a function of more than one variable by a single curve. Indeed, if fluid and bed material are kept constant then $X$ is the onl $y$ constant. In this case according to $(11)$ the variation of $1 / D$

* R. A. Bagnola (ref. [3]) did not obtain such a scatter. He based his classification of experimental points on values of $D$ and $\gamma_{s}$. In an exact "dimensional classification" the value of $T$ should also be considered, since, if the fluid used is water, ( $P=$ const, $\nu=$ const) and $w \%_{b}=0$, as in Bagnold's case, then, according to (2), $A=1$ must be $I^{-}=$ $f_{1}\left(D, \gamma_{s}, T, a_{b}\right)$ and thus in plotting $i$ versus $a_{b}$ we have $D, \gamma_{s}, T$ as parameters. However in Bagnold's case the variation in $T$ was not large and its omission did not therefore result in much scatter. 


\section{COASTAL ENGINEERING}

depends on both $Y$ and $Z$, and thus a representation of 1 as a function of $a_{b}$ only without taking into consideration the period $T$ (i.e. Y) is theoretically impossible).

The results of the measurements of ripple-lengths carrie, out in the wave channel at H.R.S., Wallingford are

shown on Fig. 3. It can be seen that the experimental points corresponding to the same constant values of $X$ and $Y$ form their own individual curves which represent the relationship $1 / D=\bar{\varphi}_{1}(Z)$. Note that the points having the same value of $X$, but different values of $Y$, form different curves, a fact which confirms experimentaliy that $I / D=\bar{\Phi}_{1}(Y, Z)$ if $X=$ const. Hence $I / D$ is in fact a function of the three variables $X, Y, Z$, as derived theoretically above.

3) CONDITIONS FOR DYNAMICAL SIMILARITY

Let $\alpha$ be any quantity;

$\alpha^{\prime}$ the prototype value of $\alpha$

$\alpha$ "the model value of $\alpha$

and $\lambda_{\alpha}=\frac{\alpha^{\prime \prime}}{\alpha^{\prime}}$ the scale of $\alpha$

The existence of dynamical similarity between the model and prototype implies

$$
\begin{aligned}
& \lambda_{\alpha} \equiv 1 \text { if } \alpha \text { is a dimensionless quantity } \\
& \lambda_{\alpha}=\text { const if } \alpha \text { is a dimensional quantity }
\end{aligned}
$$

If geometrical similarity exists, (i.e. the properties (i), (ii), (iii) are identical in model and prototype), then dynamical similarity of the two-phase phenomenon under consideration is provided by the identity of the model and prototype values of all dimensionless combinations defining the phenomenon. Thus the conditions of dynamical similarity for the two-phase phenomenon are

$$
\lambda_{X} \equiv 1 ; \lambda_{Y} \equiv 1 ; \lambda_{Z} \equiv 1 ; \lambda_{W} \equiv 1
$$

or considering (4) in terms of the scales of characteristic parameters:

$$
\left\{\begin{aligned}
\lambda_{\gamma_{s}} \cdot \lambda_{\mathrm{D}}^{3} \cdot \lambda_{p}^{-1} \cdot \lambda_{\nu}^{-2} & \equiv 1 \\
\lambda_{p} \cdot \lambda_{D} \cdot \lambda_{\gamma_{s}}^{-1} \cdot \lambda_{T}^{-2} & \equiv 1 \\
\lambda_{a_{b}} \cdot \lambda_{D}^{-1} & \equiv 1 \\
\lambda_{w_{b}} \lambda_{T} \cdot \lambda_{D}^{-1} & \equiv 1
\end{aligned}\right.
$$




\section{SIMILARITY IN SEDIMENT TRANSPORT \\ DUE TO WAVES}

There are 4 independent equations involving 7 independent scales. From a random choice of 3 scales the remaining 4 are obtained by solving (14). Thus theoretically it is always possible to obtain dynamical similarity of the two-phase phenomenon in the vicinity of the bed. However if in model and prototype the same fluid (water) is used then $\lambda_{p}=\lambda_{\nu}=1$ and the system (14) reduces into

$$
\left(\begin{array}{rl}
\lambda_{\gamma_{s}} \cdot \lambda_{D}^{3} \equiv 1 \\
\lambda_{D} \cdot \lambda_{\gamma_{s}}^{-1} \cdot \lambda_{T}^{-2} \equiv 1 \\
\lambda_{a_{b}} \lambda_{D}^{-1} \equiv 1 \\
\lambda_{w_{b}} \lambda_{T} \cdot \lambda_{D}^{-1} \equiv 1
\end{array}\right.
$$

In this case there are 4 equations involving 5 independent scales. Thus only one of them can be chosen at random.

In practice system (15) is inconvenient to use since it involves the drift velocity, wo, which must itself be estimated as a function of the properties of the wave motion. Therefore it would be more appropriate to modify (15) so that $w_{b}$ is replaced by these properties.

According to M. S. Longuet-Higgins (ref. [4]) wh can be obtained as follows

$$
w_{b}=\overline{\operatorname{cons} t} \frac{U^{2}}{C}
$$

where

$$
U=2 \frac{a_{b}}{T}
$$

and

$$
C=\frac{L}{T}
$$

Thus

$$
w_{b}=\text { const } \frac{a_{b}^{2}}{T L}
$$

and therefore

$$
\lambda_{w_{b}}=\lambda_{a_{b}}^{2} \lambda_{T}^{-1} \lambda_{L}^{-1}
$$

Consider that $T, I$ and $h$ are connected by the C-formula in following form

$$
\frac{c^{2}}{g L}=\frac{L}{g T^{2}}=\Psi\left(\frac{h}{L}\right)
$$




\section{COASTAL ENGINEERING}

where:

$$
\Psi\left(\frac{h}{L}\right)=\frac{1}{2 \pi} \tanh 2 \pi \frac{h}{L}
$$

This is an exact expression for the theory of "waves with small amplitude" and represents the first order of approximation for the "waves with finite amplitude" (Stokes). Assumin! $\lambda_{\mathrm{g}}=1$, we obtain from (21)

$$
\lambda_{L}=\lambda_{\Psi} \lambda_{T}^{2}
$$

Considering (20) and (23), the system (15) can be expressed as follows:

$$
\left(\begin{array}{ll}
\lambda_{\gamma_{b}} \lambda_{D}^{3} \equiv 1 \\
\lambda_{D} & \lambda_{\gamma_{S}}^{-1} \lambda_{T}^{-2} \equiv 1 \\
\lambda_{a_{b}} \cdot \lambda_{D}^{-1} \equiv 1 \\
\lambda_{a_{b}}^{2} & \lambda_{D}^{-1} \cdot \lambda_{L}^{-1} \equiv 1 \\
\lambda_{L} \cdot \lambda_{T}^{-2} \equiv \lambda_{\Psi}
\end{array}\right)
$$

with

$$
\lambda_{\Psi}=\frac{\tanh 2 \pi h^{\prime \prime} / L^{\prime \prime}}{\tanh 2 \pi h^{\prime} / L^{\prime}}=\frac{\tanh \left[2 \pi h^{\prime} / L^{\prime}\left(\lambda_{h} / \lambda_{L}\right)\right]}{\tanh 2 \pi h^{\prime} / L}
$$

or for small prototype values $h^{\prime} / L^{\prime}$ simply:

$$
\lambda_{\Psi} \approx \frac{\lambda_{h}}{\lambda_{L}}
$$

Thus the value $\lambda_{\psi}$ depends on the prototype value, $h^{\prime} / I^{\prime}$, and the distortion, $\lambda_{h} / \lambda_{I}$. The system (24) consists of 5 equations involving 6 independent scales. Hence only one of them can be chosen at random.

\section{DISCUSSION}

a) UNDISTORTED MODEL: ( $\lambda_{h}=\lambda_{L}$, i.e. $\left.\lambda_{\Psi}=1\right)$

Substituting $\lambda_{\Psi}=1$ the system (24) gives unity for all scales. Thus by using the same fluid in model and prototype dynamical similarity of the two-phase phenomenon on an undistorted model is impcssible. 


\section{SIMILARITY IN SEDIMENT TRANSPORT \\ DUE TO WAVES}

If the influence of drift (the condition $\lambda_{w} \equiv 1$ ) can be neglected, i.e. if the fourth equation in (24) is rejected, then the solution of (24) gives

$$
\left(\begin{array}{rl}
\lambda_{b} & =\lambda_{a_{b}} \\
\lambda_{\gamma_{s}} & =\lambda_{a_{b}}^{-3} \\
\lambda_{T} & =\lambda_{a_{b}}^{2} \\
\lambda_{h}= & \lambda_{L}=\lambda_{a_{b}}^{4}
\end{array}\right.
$$

This is a Froudian model $\left(\lambda_{T}=\sqrt{\lambda_{L}}\right)$, where the bed material is heavier but finer than in prototype. The velocity scales in this case are:

$$
\lambda_{w_{b}}=\lambda_{a_{b}}^{-4} ; \quad \lambda_{v}=\lambda_{a_{b}}^{-1} ; \quad \lambda_{c}=\lambda_{a_{b}}^{2}
$$

b) DISTORTED MODEL (DRIFT CONDITION IS SATISFIED) $\left(\lambda_{\psi} \neq 1\right)$

Preserving the fourth equation and assuming $\lambda_{\psi} \neq 1$ the solution of (24) gives

$$
\left(\begin{array}{l}
\lambda_{D}=\lambda_{a_{b}} \\
\lambda_{\gamma_{s}}=\lambda_{a_{b}}^{-3} \\
\lambda_{T}=\lambda_{a_{b}}^{2} \\
\lambda_{L}=\lambda_{a_{b}} \\
\lambda_{\Psi}=\lambda_{a_{b}}^{-3}
\end{array}\right)
$$

selecting $\lambda_{L}=\lambda_{a_{b}}$ we can obtain all the other scales including $\lambda_{\psi}=\lambda_{a_{b}}^{-3}$ i.e.

$$
\frac{\tanh \left[2 \pi h^{\prime} / L^{\prime}\left(\lambda_{h} / \lambda_{L}\right)\right]}{\tanh 2 \pi h^{\prime} / L^{\prime}}=\lambda_{L}^{-3}
$$

Hence, knowing $\lambda_{L}$ and the prototype value $h^{\prime} / L^{\prime}, \lambda_{h}$ is obtained as the solution of the transcendent equation above. The model is not Froudian $\left(\lambda_{T}=\lambda_{L}^{2}\right)$ and the bed material consists of larger but lighter grains than in the prototype. Indeed if $\lambda_{h}<1$ then (30) gives $\lambda_{L}>1$, and thus $\lambda_{a_{b}}>1$ and consequently $\lambda_{D}>1$ and $\lambda_{\gamma_{s}}<1$. Obviously this non-Froudian model in which $\lambda_{L}>1$ and ${ }^{2} \lambda_{T}>1$ is not as convenient for practical purposes as the undistorted model discussed in (a): it therefore represents only a theoretical solution of the problem. 
COASTAL ENGINEERING
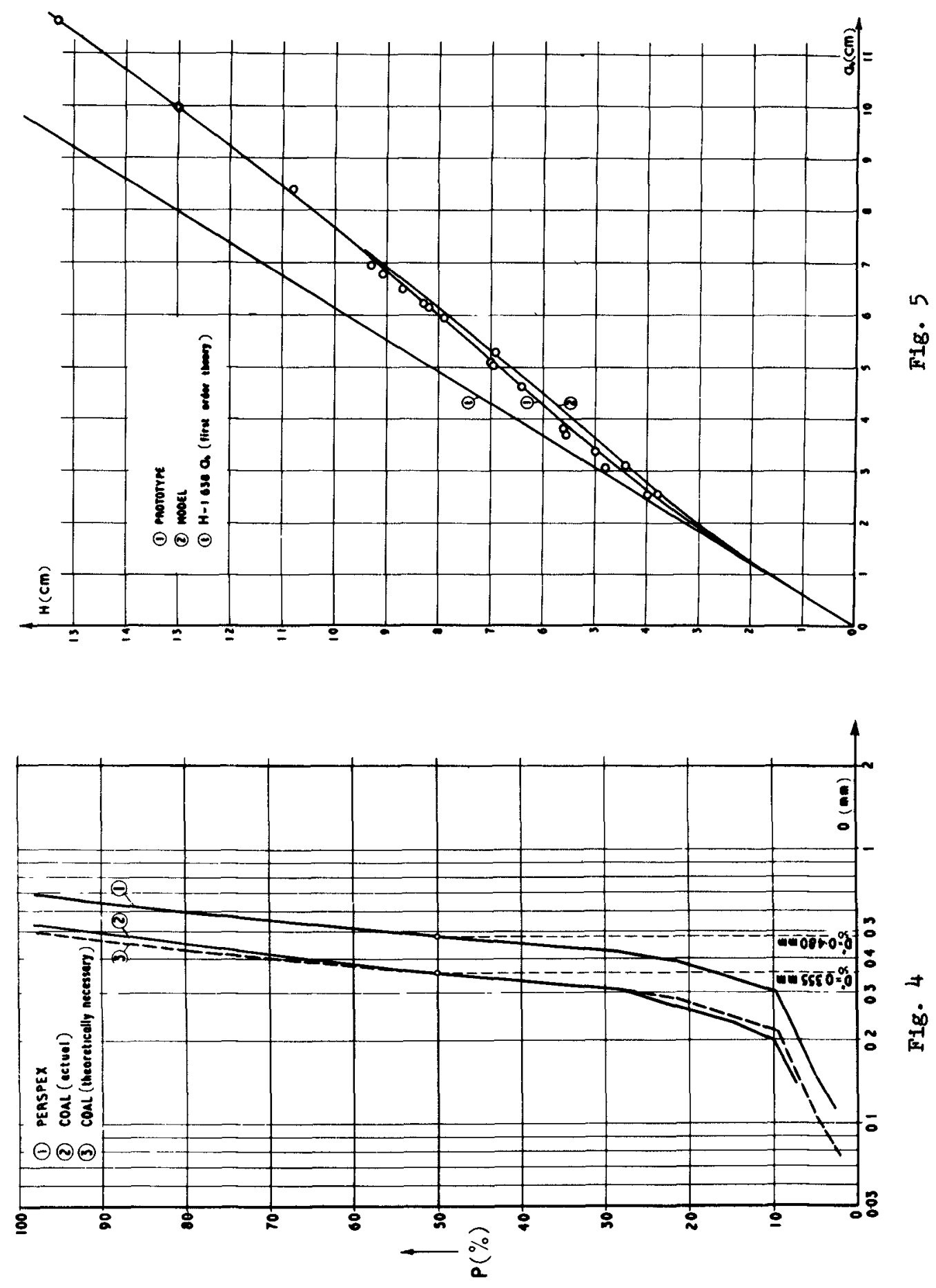


\section{SIMILARITY IN SEDIMENT TRANSPORT \\ DUE TO WAVES}

\section{4) THE EXPERIMENTAL RESULTS}

In order to confirm the theoretical considerations stated above, experiments were carried out in the $75 \mathrm{~cm}-w i d e$ wave channel at H.R.S., Wallingford. The measurements were made on a Froudian model (as described in 3(a)). It has to be proved that in such a model the conditions for dynamical similarity at the bed are

$$
\lambda_{X} \equiv 1 ; \lambda_{Y} \equiv 1 ; \lambda_{Z} \equiv 1
$$

(assuming of course that (i), (ii) and (iii) are specified). If, in fact $X, Y, Z$ represent the "complete set" of dimensionless variables, then the conditions

$$
\begin{aligned}
& \mathrm{X}^{\dagger} \equiv \mathrm{X}^{\prime \prime} \\
& \mathrm{Y}^{\dagger} \equiv \mathrm{Y}^{\prime \prime} \\
& \mathrm{Z}^{\prime} \equiv \mathrm{Z}^{\prime \prime}
\end{aligned}
$$

must make all corresponding dimensionless functions, $\varphi_{A}^{\prime}$ and $\varphi_{A}^{\prime \prime}$, identical

$$
\varphi_{A}^{\prime}=\varphi_{A}\left(X^{\dagger}, Y^{\prime}, Z^{\prime}\right)=\varphi_{A}\left(X^{\prime \prime}, Y^{\prime \prime}, Z^{\prime \prime}\right)=\varphi_{A}^{\prime \prime}
$$

with the scale of $A$

$$
\lambda_{A}=\lambda_{D}^{-x} \cdot \lambda_{\gamma_{S}^{y}}^{-y} \cdot \lambda_{T}^{-z}
$$

Perspex $\left(\gamma_{s}^{\prime}=0,19\right)$ was chosen as bed material for the prototype, and coal $\left(\gamma_{s}^{\prime \prime}=0.48\right)$ for the model. Thus

$$
\lambda_{\gamma_{s}}=\frac{\gamma_{s}^{\prime \prime}}{\gamma_{s}^{\prime}}=2.528
$$

According to the first and second equations of (27)

$$
\lambda_{D}=\frac{1}{\sqrt[3]{\lambda_{\gamma_{s}}}}=0.735
$$

Fig. 4 shows the particle-size distribution curves of the perspex and coal used in the experiments. In order to satisfy the geometrical condition, (ii), the coal was prepared so as to have a particle-size distribution curve similar to that for the perspex. As shown in Fig. 4 the actual ratio $\lambda_{\mathfrak{D}}$ was

$$
\lambda_{\mathrm{D}} \cong \lambda_{\mathrm{D}_{\mathrm{s} 0}}=\frac{0.355}{0.48}=0.74
$$

correspondingly the system (27) yields: 


\section{COASTAL ENGINEERING}

$$
\begin{aligned}
\lambda_{a_{b}} & =\lambda_{d}=0.74=1 / 1.35 \\
\lambda_{T} & =0.74^{2}=0.548=1 / 1.82 \\
\lambda_{h}=\lambda_{L} & =0.74^{4}=0.301
\end{aligned}
$$

Selecting the prototype values

and consequently $\mathrm{L}^{\prime}=399 \mathrm{~cm}=$ const

$$
\left.\begin{array}{l}
\mathrm{T}^{\prime}=1.82 \mathrm{~s}=\text { const } \\
\mathrm{h}^{\prime}=65.0 \mathrm{~cm}=\text { const } \\
\mathrm{L}^{\prime}=399 \mathrm{~cm}=\text { const }
\end{array}\right\}
$$

the corresponding model values were

$$
\left.\begin{array}{l}
T^{\prime \prime}=1.00 \mathrm{~s}=\text { const } \\
\mathrm{h}^{\prime \prime}=19.6 \mathrm{~s}=\text { const } \\
\mathrm{L}^{\prime \prime}=120 \mathrm{~cm}=\text { const }
\end{array}\right\}
$$

Therefore:

$$
X^{\prime}=206,13=\text { const } ; Y^{\prime}=77,75 \cdot 10^{-6}
$$

and

$$
X^{\prime \prime}=210,67=\text { const } ; Y^{\prime \prime}=75,39 \cdot 10^{-6}(40)
$$

Since $\frac{X^{\prime}}{X^{\prime \prime}}=97,84 \%$ and $\frac{Y^{\prime \prime}}{Y^{\prime}}=96,65 \%$ it can be assumed that the conditions $X^{\prime}=X^{\prime \prime}$ and $Y^{\prime}=Y^{\prime \prime}$ are satisfied from the point $c$ view of the accuracy of measurement. Since $a_{b}$ (i.e. Z) was the only variable, the experimental points for both model anc prototype must lie on the same curve $\left(\Pi_{A}=\overline{\bar{\varphi}}_{A}(z)\right)$ in the dimensionless system of coordinates for any related mechanice quantity $A$. This statement was proved experimentally for 3 independent aspects of the phenomenon $\mathrm{A}_{1}, \mathrm{~A}_{2}, \mathrm{~A}_{3}$ (which, according to the theory of dimensions is sufficient in order to prove the existence of dynamical similarity).

$$
\begin{aligned}
& A_{1}= 1 \text { ripple length } \\
& A_{2}=\Delta \text { ripple height } \\
& A_{3}= \text { the total weight of the bed material trans- } \\
& \text { ported during the certain time t from a tray } \\
& \text { inserted in the bed. }
\end{aligned}
$$

Tl-values of these quantities are respectively

$$
\Pi_{1}=\frac{1}{D} \quad \Pi_{\Delta}=\frac{\Delta}{D} \quad \Pi_{G}=\frac{G}{\gamma_{s} D^{3}}
$$




\section{SIMILARITY IN SEDIMENT TRANSPORT DUE TO WAVES}

The measurement of the orbit lengths $a_{b}$ was made by means of a pendulum which consisted of a disc which was weightless in water, moving on a spanned steel string parallel to a ruler. For the constant prototype values $h=65 \mathrm{~cm}$ and $T=1,82 \mathrm{~s}$ this instrument gave the relationship between $a_{b}$ and $H$ as shown in Fig. 5 by curve (1). As seen from Fig. 5, as H increases this experimental curve systematically deviates from the theoretical curve $(T)$ ( $(T)$ representing the linear relation between $a_{b}$ and $H$ for waves of small amplitude). It was assumed that the ratio $\mathrm{ab} / \mathrm{H}$ given by the curve (1) is valid for all waves of the same $L / h$. Therefore the orbit lengths in the model were obtained from curve (2) (drawn homologous to curve $(1)$ to the scale $\lambda_{a_{b}}=0.74$ ) by measuring the wave heights $\mathrm{H}$.

For the measurements $I$ and $\Delta$ the bed of the flume $(8.00$ $\mathrm{x} 0.75 \mathrm{~m}^{2}$ ) was covered with material to a depth of not less than $3 \mathrm{~cm}$. The values $I$ and $\Delta$ were obtained as mean values of a "ripple train" (consisting of at least 20 ripples) which was traced from the central observation window on the side of the flume.

The total weight $G$ leaving the tray (Fig. 6) during the time intervals $t^{*}=15 \mathrm{~min}$ and $t^{\prime \prime}=8.25 \mathrm{~min}$ in prototype and model respectively was measured as

$$
G=G_{q}+G_{r}
$$

The results of measurements of ripple length in model and prototype are shown in Figs. 7 and 8 . The dimensional plotting in Fig. 7 shows that the experimental points of prototype and model form two homologous curves to the scale $\lambda_{a_{b}}=$ 0.74 ; whereas both prototype and model points form the same curve $I / D=\bar{\Phi}_{1}(Z)$ in the dimensionless plotting in Fig. 8 .

In Fig. 11 the dimensionless ripple heights $\Delta / D$ are plotted. Once again there is a tendency for both model and prototype experimental points to lie on the same curve $\Delta / D=\bar{\phi}_{\Delta}(Z)$. (Note that $I / D$ is always an increasing function of $z$ whereas $\Delta / D$ tends to zero at a certain value of $Z$.) Thus the ripples disappear because their height, and not their length, tends to zero.

Figs, 10 and 11 show the results of measurements of the total weight of bed material transported from geometrically similar trays during equivalent periods of time. In this case also, the experimental points form homologous curves (parallel 
COASTAL ENGINEERING

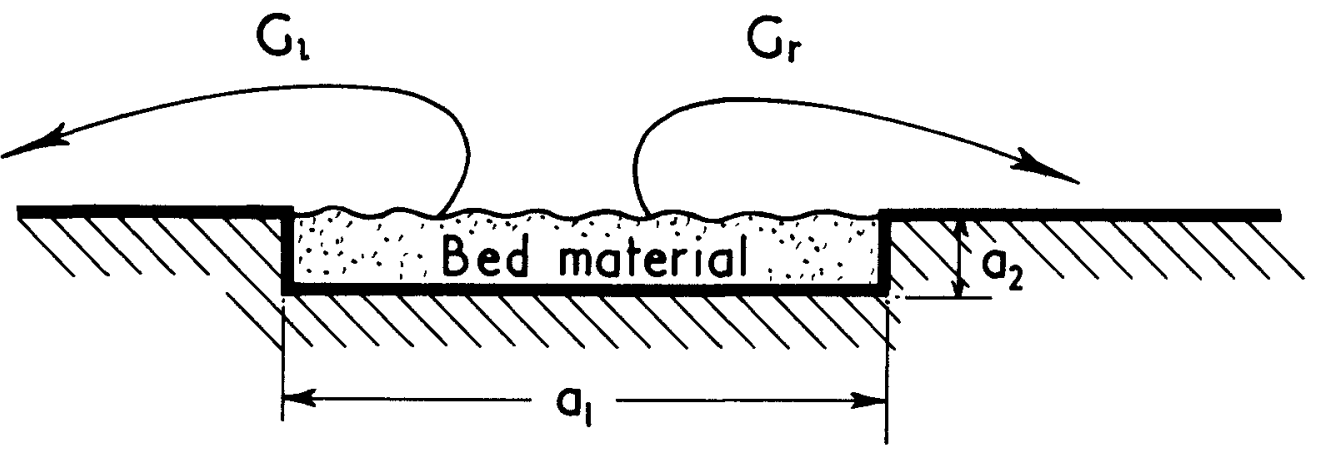

$$
\begin{aligned}
& a_{1}^{\prime}-1.50 \mathrm{~m} ; a_{2}^{\prime}-3 \mathrm{~cm} \quad \text { (Prototype) } \\
& a_{1}^{\prime \prime}=1.11 \mathrm{~m}, a_{2}^{\prime \prime}=2.2 \mathrm{~cm} \text { (Model) }
\end{aligned}
$$

FIg. 6

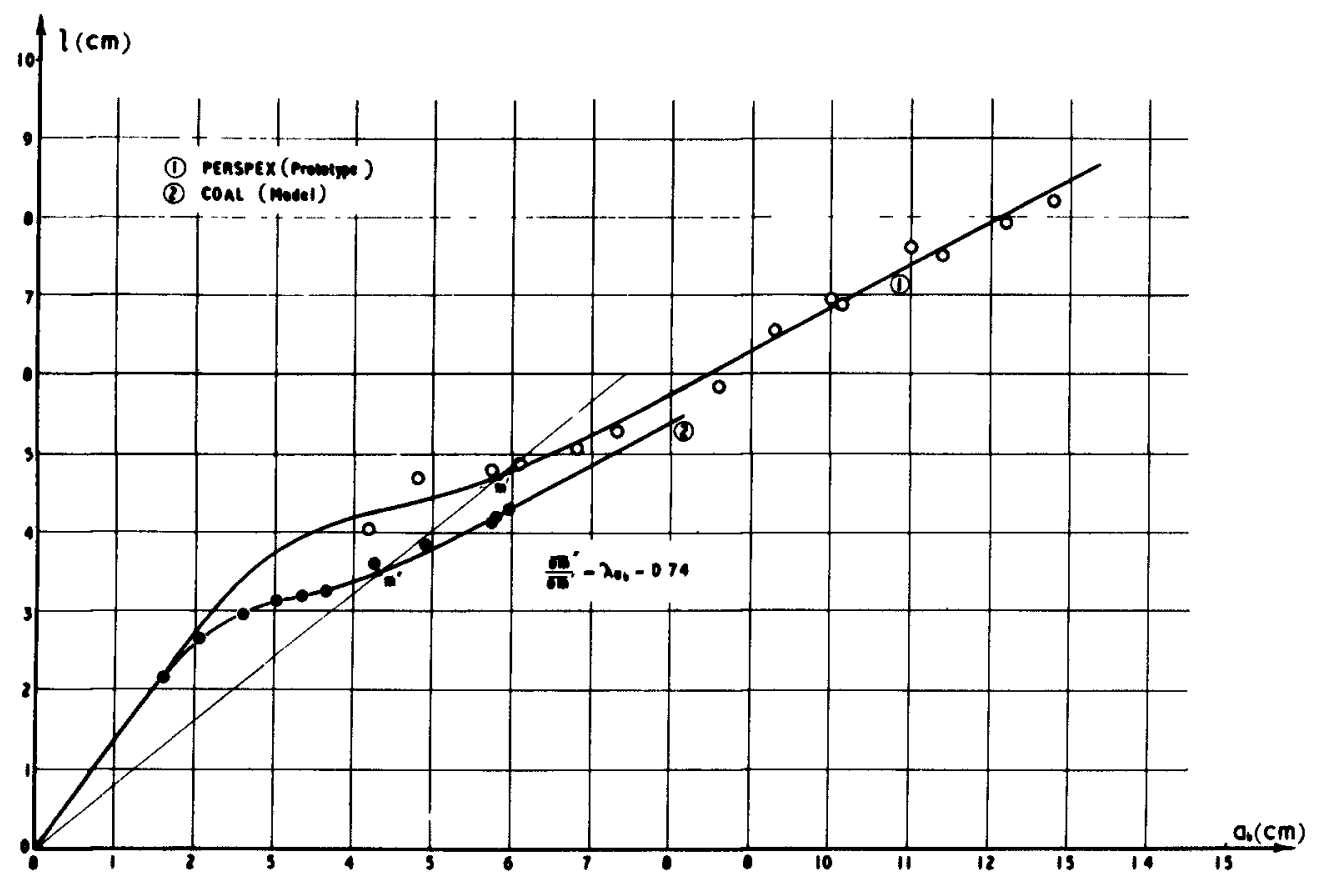

Fig. 7 
SIMILARITY IN SEDIMENT TRANSPORT

DUE TO WAVES
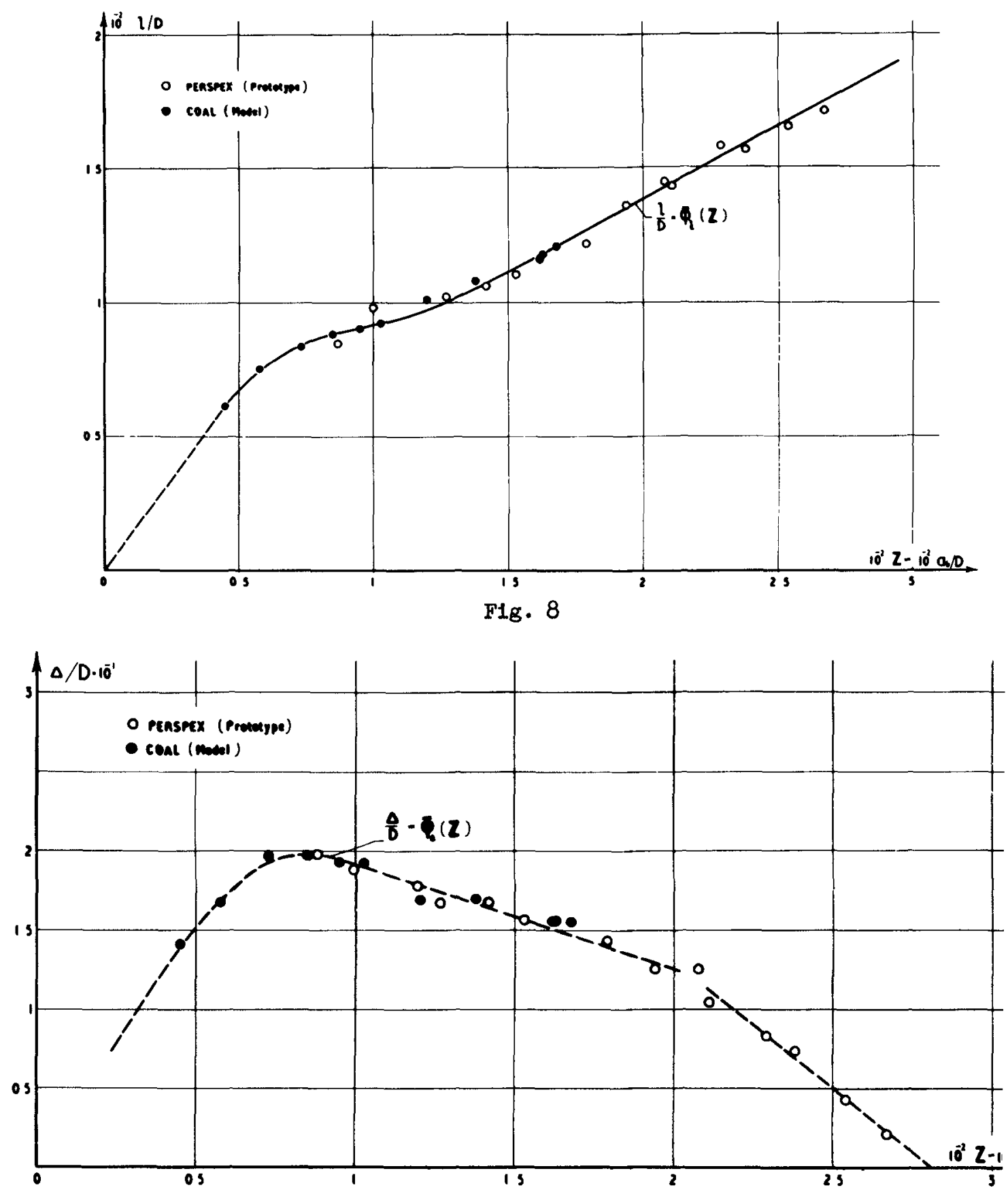

Fig. 9 
COASTAL ENGINEERING

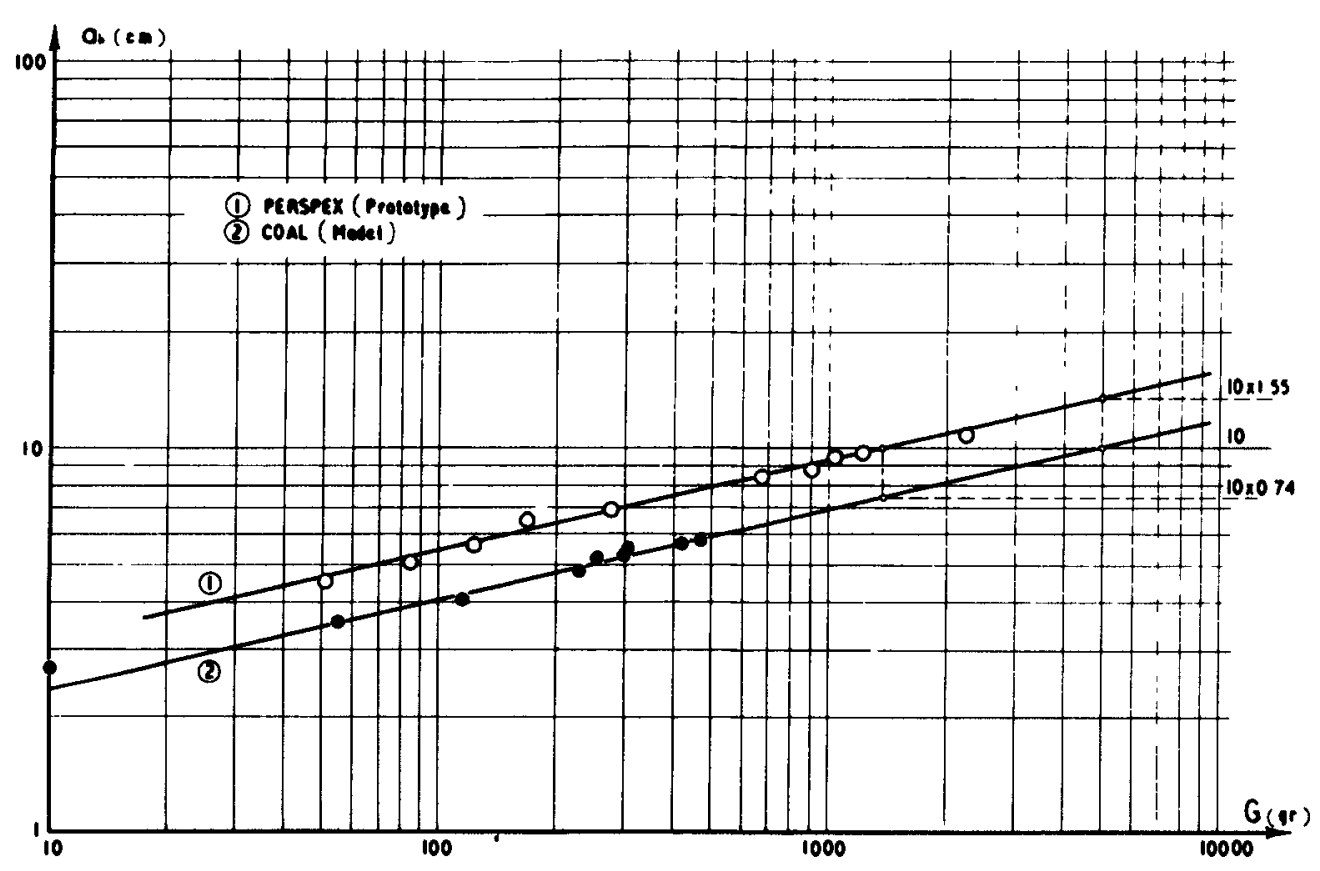

F18. 10

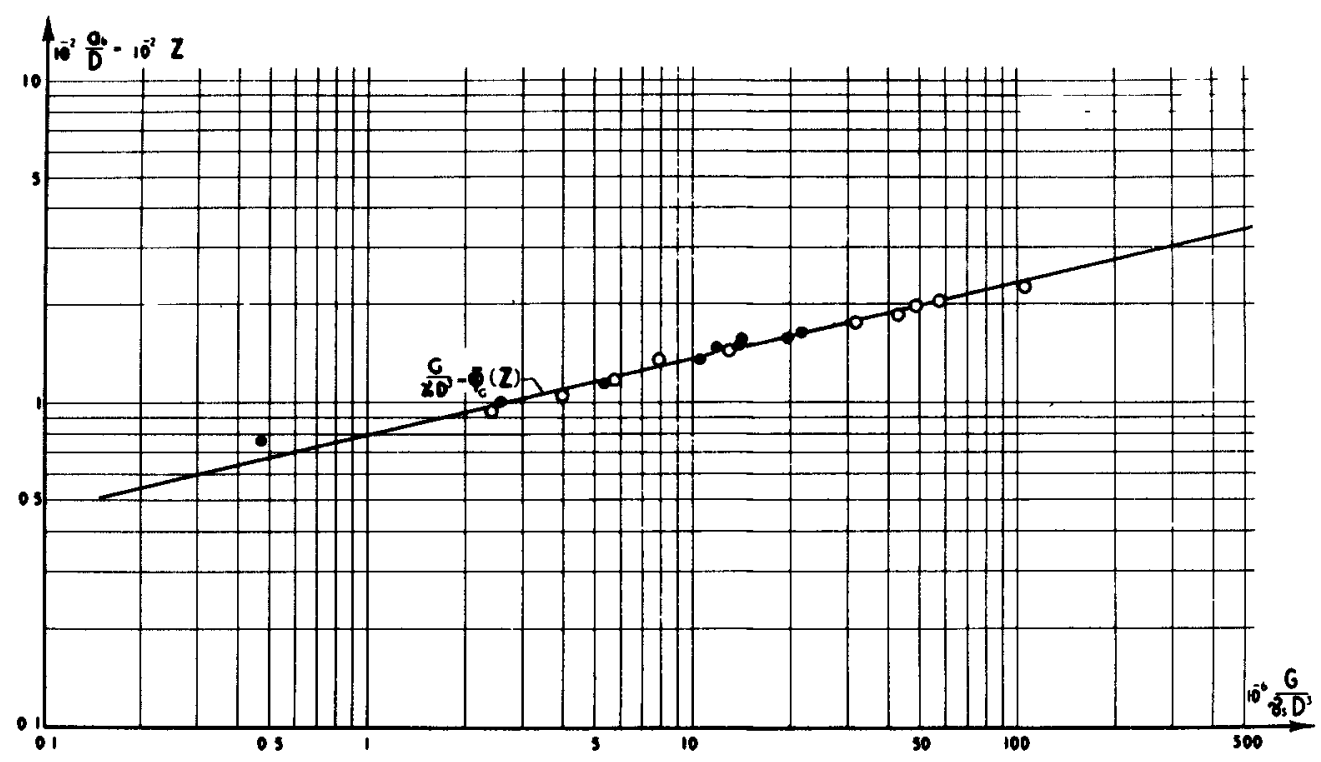

FIg. 11 


\section{SIMILARITY IN SEDIMENT TRANSPORT \\ DUE TO WAVES}

straight lines with log-log coordinates) to the scale $\lambda_{a_{b}}=$ 0.74 in the dimensional plotting in Fig. 10; and a single curve (straight line) with logarithmic coordinates in the dimensionless representation in Fig. 11.

This study was carried out as part of the research programme of the Hydraulics Research Board of the Department of Scientific and Industrial Research, and is published with the permission of the Director of Hydraulics Research.

\section{REFERENCES}

Sedov, I. I. (1959). "Similarity and Dimensional Methods in Mechanics" London: Infosearch Ltd.,

Inman, D. (1957) . "Wave-generated ripples in nearshore sand" T.M. 100, U.S. Beach Erosion Board.

Bagnold, R. A. (1946). "Motion of waves in shallow water, Interaction between waves and sand bottoms". Proceedings of the Royal society, A, vol 187.

Longuet-Higgins, M. S. (1957). "The mechanics of the boundary-layer near the bottom in a progressive wave", Proceedings of sixth conference on Coastal Engineering, Chapter 10 - Appendix.

Manohar, M. (1955). "Mechanics of bottom sediment movement due to wave action", T.M. 75, U.S. Beach Erosion Board. 